\title{
Evaluation of Self-Care Practice and Its Associated Factors in Adult Diabetic Patients, Ayder Diabetic Clinic, Mekelle, Ethiopia
}

Migbnesh Weledegebriel (iD) Afework Mulugeta ${ }^{2}$

Abraha Hailu'

'Department of Internal Medicine, School of Medicine, College of Health Sciences, Mekelle University, Mekelle, Tigray, Ethiopia; ${ }^{2}$ Department of Public Health, School of Public Health, College of Health Sciences, Mekelle University, Mekelle, Tigray, Ethiopia
Correspondence: Migbnesh Weledegebriel Tel +251914126128

Email migbey12@gmail.com
Background: Self-care activities in patients with diabetes mellitus are behaviors undertaken by people with or at risk of diabetes in order to successfully manage the disease on their own. Even though self-care practice plays a critical role in preventing as well as delaying diabetesrelated complications, there is poor self-care practice by patients with diabetes mellitus. In Ethiopia, especially in Tigray, there are few studies assessing self-care practice of patients with diabetes mellitus and thus this study will have an input in this area.

Objective: To evaluate self-care practice and its associated factors in adult patients with diabetes mellitus having follow-up in Ayder Comprehensive Specialized Hospital, Diabetes Clinic.

Methods: A hospital-based cross-sectional analysis was made from March to May, 2015. A total of 410 patients with diabetes mellitus were included. The study participants were selected by a systematic random sampling method. Characteristics of study participants were analyzed using descriptive statistics, while bivariate and multivariable logistic regression was used to identify the association between dependent and independent variables.

Results: Two hundred and eighty-eight (70.2\%) study participants were above 30 years of age and $254(62.9 \%)$ study participants were males. More than half $(52.9 \%)$ of the study participants were type-2 diabetes patients. This study showed $207(50.5 \%)$ had poor diabetes self-care practice. In multivariate logistic regression, low income $(\mathrm{AOR}=0.518,95 \% \mathrm{CI}$ : $0.288-0.929$ ) and poor knowledge about diabetes (AOR $=5.026,95 \%$ CI: 2.893-8.734) were found to be significantly associated with poor self-care practice.

Conclusion: The proportion of poor self-care practice was high, implying the need for regular follow-up as an integral component of the long-term management of diabetes mellitus.

Keywords: self-care practice, diabetes mellitus

\section{Introduction}

\section{Background}

Diabetes mellitus (DM) is defined as a collection of common metabolic disorders that share the phenotype of hyperglycemia. Factors contributing to hyperglycemia include reduced insulin secretion, decreased glucose utilization, and increased glucose production. DM is the leading cause of end-stage renal disease (ESRD), non-traumatic lower extremity amputations, adult blindness and cardiovascular diseases. The worldwide prevalence of DM has risen dramatically over the past two decades, from an estimated 30 million cases in 1985 to 285 million in $2010 .^{1}$ 
The International Diabetes Federation (IDF) estimates that there are 382 million people in the age group of 20-79 years living with diabetes in 2013 and $80 \%$ of them live in low and middle income countries. Among those, $46 \%$ of the people with diabetes are undiagnosed. Currently, the region with the highest number of people with diabetes is the Western Pacific, while Africa has the lowest number. Globally, the prevalence of diabetes is estimated to increase to 592 million people by 2035 and it will double in Africa. The prevalence of diabetes in Africa in 2013 was $5.7 \%$ according to the IDF and different studies show a prevalence range of $1-12 \%$ in sub-Saharan African countries. The prevalence of DM in Ethiopia in adults in the age group between 20-79 years of age is $4.36 \%$ (1.9 million), of which $75 \%$ of the patients are undiagnosed. $^{2}$

Despite regular follow-up and medication, diabetic patients may have high blood sugar; this is the condition that points to self-care where diabetic individuals take measures to maintain their wellbeing. ${ }^{3}$ In diabetic patients, the aim of treatment is to avoid or reduce acute or chronic diabetes related complications. This decreases serious morbidity and mortality, and loss of productiveness, by adhering to proper self-management that includes blood glucose monitoring, drug adherence, regular exercise, sticking to recommended diet and other lifestyle modifications. ${ }^{4}$

Patients with diabetes who practice sufficient selfmanagement have shown better outcomes (live longer, enjoy a higher quality of life, and suffer fewer symptoms and minimal complications). In contrast, patients who have inadequate diabetic self-management have impacts on their morbidity and mortality, it increases the costs of medication and laboratory tests and costs in time and effort of care providers. ${ }^{5}$ From a study done among patients with diabetes on follow-up to assess diabetic self-care, nearly half of the patients had reported poor knowledge about diabetes and self-care practice, which results in poor selfcare activities on exercise, intake of healthy diet, adhering to prescribed medication and self-monitoring of blood glucose level. In this study, age, monthly income, occupation, years of suffering from diabetes, educational status, and knowledge level were the independent predictors of self-care practice. ${ }^{6}$ A study done in Felege Hiwot Hospital, Northwest Ethiopia, revealed low levels of knowledge and practice among DM patients. Age, monthly income, educational status and duration of DM therapy were associated with good knowledge and practice of participants.
Improving knowledge and practice of diabetic patients through active education is advisable. Involvement of both governmental and non-governmental organizations is also crucial to help patients receive maximum benefit from the health-care service. ${ }^{7}$

The incidence and prevalence of both type- 1 and type$2 \mathrm{DM}$ is increasing in developing countries with changes in lifestyle. Most of these patients present to hospitals with complications from both known and newly diagnosed diabetes which could have been prevented by providing basic diabetic education at health centers or hospitals. Studies have shown that by educating patients and families on how to perform diabetic self-care, good glycemic control and reduction in acute as well as chronic complications has been achieved, compared with those who are not provided with this information. ${ }^{8,9}$

In our hospital there is a diabetes clinic but diabetes self-care education is given inconsistently. Hence, this study will have an input for improvement of health education and counseling of diabetic patients to prevent diabetic complications in Ayder Referral Hospital and the country as a whole. A conceptual framework of factors affecting diabetic self-care, developed by the main investigator, is shown in Figure 1.

\section{Objectives of the Study General Objective}

To evaluate self-care practice and its associated factors in adult diabetic patients having follow-up in Ayder Comprehensive Specialized Hospital (ACSH), Diabetes clinic.

\section{Specific Objectives}

- To determine the magnitude of self-care practice in adult diabetic patients.

- To determine the predictors of self-care practice in adult diabetic patients.

\section{Methods and Materials}

\section{Study Area}

The study was conducted in Ayder Comprehensive Specialized Hospital (ACSH), Mekelle, Tigray which is found $778 \mathrm{~km}$ to the north of Addis Ababa. It started as a referral and specialized medical center in 2008 serving 8 million people. Internal medicine is one of the major departments in the hospital and it has different specialty clinics. The diabetes outpatient department (OPD) is one of the services it provides, which started in April 2011. In 


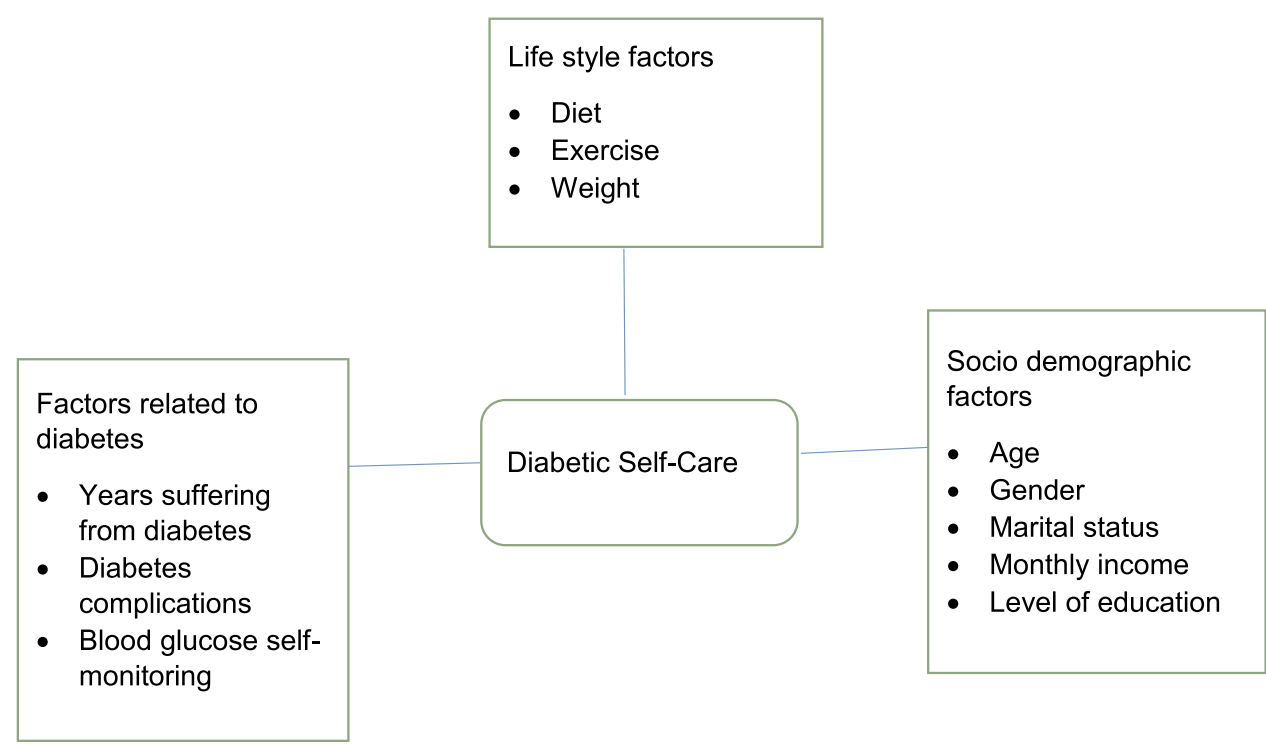

Figure I Conceptual framework of factors affecting diabetic self-care, developed by the principal investigator.

this clinic there are three nurses, one medic and three internal medicine specialists interested to subspecialize in endocrine and metabolic diseases. Every day in the afternoon the nurses give diabetic education to patients using posters and talks. In addition to this, patients gather monthly which is organized by the Ethiopian Diabetic Association for education and experience sharing among themselves.

\section{Study Design and Period}

The study design is an institution-based cross-sectional study complemented with secondary data from patient records. The study was conducted between March 2015 and May 2015.

\section{Source Population}

All adult diabetic patients who have follow-up in the diabetes clinic in ACSH.

\section{Study Population}

All adult diabetic patients who visited ACSH for their follow-up during the data collection period.

\section{Inclusion and Exclusion Criteria Inclusion Criteria}

Age more than 15 years who have been on follow-up for at least six months and above at the diabetes clinic of ACSH were included.

\section{Exclusion Criteria}

Participants who did not give their consent.

\section{Sample Size Determination and Sampling Methods \\ Sample Size Determination}

The minimum sample size required for the study was estimated using the single population formula

$$
\mathrm{N}=\frac{Z \frac{\alpha^{2}}{2} P(1-P)}{d^{2}}
$$

where

$$
\mathrm{N}=\text { sample size }
$$

$\mathrm{Z}=$ is the standard normal deviation, set at 1.96 (for $95 \%$ confidence interval)

$\mathrm{D}=$ desired degree of precision (taken as 0.05 )

$\mathrm{P}=$ estimate of our target population having poor diabetic self-care practice of $45 \%$ from Nekemte Referral Hospital. ${ }^{6}$

Adjustment for finite population correction and a 10\% rate of non-responses and invalid responses yielded a final sample size of 410 .

\section{Sampling Procedure}

The recorded diabetic patients from the diabetic unit was used as a sampling scheme for randomization. Every fifth patient was selected using a systematic random sampling method.

\section{Variables \\ Independent variables}

The independent variables included were age, sex, marital status, occupation, educational status, income, knowledge about diabetes and duration of diabetes diagnosis. 


\section{Dependent Variable}

The dependent variable was diabetic self-care practice.

\section{Data Collection Procedure}

Data were collected by a structured interviewer administered questionnaire to evaluate diabetic self-care practice in adult patients with diabetes who have medical checks in the diabetes clinic in ACSH. The questionnaire was first developed in English and then converted into Tigrigna that can easily be understood by the study participants. The questionnaire was pretested in non-communicable disease follow-up clinic of Mekelle Hospital.

Check lists were prepared to take secondary data such as FBS and weight/height measurements done at the time of enrollment, and HgbAlc determination within a 3-months period from patient chart.

\section{Data Quality Assurance}

To assure the quality of data the appropriately designed structured questionnaire was used. Pre-test was conducted in Mekelle Hospital. Based on the findings of the pre-test, some questions were modified. Every day the collected data were reviewed and checked for completeness and consistency of the response.

\section{Data Analysis}

Data were cleaned, edited and entered into Epi data version 3.1 and analyzed by using SPSS version 20 . Characteristics of study participants were analyzed using descriptive statistics such as frequency and percentage for categorical data. Bivariate and multivariate logistic regression was used to identify the association between dependent and independent variables. All variables with $\mathrm{p}$ value $\leq 0.25$ at bivariate logistic regression analysis were fitted into multivariable logistic regression to identify the determinants of poor self-care practice. Statistical significance was considered at a $\mathrm{P}$ value of $<0.05$. Odds ratio with $95 \%$ CI was used to show the strength of association between independent and dependent variables.

\section{Operational Definition}

Self-care: A collection of activities that diabetic individuals perform on their own to control and maintain their disease, health and well-being.

Self-care practice: diabetic self-care practice was assessed by dietary habit, regular exercise, blood glucose monitoring and self-examination of the feet. Of these parameters, diet, blood glucose monitoring and regular exercise were taken as mandatory; self-examination of feet was considered as optional.

- Good self-care practice - a study participant who fulfills the mandatory and/or optional criteria.

- Poor self-care practice - a study participant who does not fulfill any of the mandatory and/or optional criteria.

Knowledge on diabetes - includes lifestyle modification, prevention of complications, hyperglycemia or hypoglycemia symptoms, and diabetes definition.

Good knowledge patients who scored $\geq 50 \%$ of the above criteria.

Poor knowledge patients who scored $<50 \%$ of the above criteria.

\section{Results}

\section{Socio-Demographic Characteristics}

Out of the total 420 diabetic patients, 410 of them had completed the study, which gave a response rate of $97.6 \%$. The majority of the study participants (70.2\%) were above 30 years of age and $29.8 \%$ were in the age group of $15-30$ years; $62 \%$ of study participants were males and $62.9 \%$ were married. Over a third (36.1\%) had no formal education while $21.2 \%$ and $16.6 \%$ of the participants had completed grades 8 and 12, respectively. Nearly $74 \%$ of respondents were urban residents (Table 1).

\section{Clinical Characteristics of Participants and Lifestyle Related Information}

Just over half $(52.9 \%)$ of the respondents had type-2 diabetes, $47.1 \%$ had type- 1 diabetes and $70.7 \%$ had diabetes with duration of less than 5 years; $4.6 \%$ had family history of diabetes. Among the respondents, $44.1 \%$ had FBS of between $70-140 \mathrm{mg} / \mathrm{dl}$. About $8.8 \%$ of the participants had FBS $>250 \mathrm{mg} / \mathrm{dl}$. A majority of the study participants $(72.9 \%)$ had a BMI of $18.5-24.9$, while $16.1 \%$ had BMI $>25$. Nearly $60 \%$ of the study participants regularly exercise. The type of exercise was aerobic exercise in $53.2 \%$ of the study participants (Table 2 ).

\section{Prevalence of Diabetic Self-Care Practices}

In this study, diabetic self-care practice was assessed by dietary habit, regular exercise, blood glucose monitoring, and self-examination of the feet. Among the 
Table I Socio-Demographic Characteristics of Diabetic Patients from the Diabetes Clinic of ACSH, Tigray, Ethiopia, 2015 $(\mathrm{N}=410)$

\begin{tabular}{|l|l|l|l|}
\hline \multicolumn{2}{|l|}{ Variable } & Frequency & Percentage \\
\hline Age & $15-30$ & 122 & 29.8 \\
& $>30$ & 280 & 70.2 \\
\hline Sex & Female & 156 & 38 \\
& Male & 254 & 62 \\
\hline Residence & Rural & 107 & 26.1 \\
& Urban & 303 & 73.9 \\
\hline Occupation & Governmental & 93 & 22.7 \\
& employee & & \\
& Merchant & 91 & 22.2 \\
& Farmers & 136 & 33.2 \\
& Housewife & 57 & 13.9 \\
& Others* & 33 & 8.0 \\
\hline Marital status & Single & 106 & 25.9 \\
& Married & 258 & 62.9 \\
& Others ** & 46 & 11.3 \\
\hline Educational & Informal education & 148 & 36.1 \\
status & I-12th grade & 155 & 37.8 \\
& Above secondary & 107 & 26.1 \\
\hline Yearly income & $<15,000$ & 279 & 68 \\
& $>15,000$ & 131 & 32 \\
\hline
\end{tabular}

Notes: a. *Students, non-governmental, maid. b. **Divorced, widowed. c. ***Ethiopian birr.

respondents $210 \quad(51.5 \%)$ had regular blood sugar checkup per visit and only $69(15.8 \%)$ of participants reported self-examination of their feet. The prevalence of poor self-care practice in this study was found to be 207 (50.5\%) (Table S1).

\section{Factors Influencing Diabetic Self-Care}

\section{Practices}

Binary logistic regression was conducted and variables with a P-value $<0.25$ were considered for multivariable logistic regression. On bivariate analysis, sex, age, marital and educational status, occupation, current residency, income, knowledge of diabetes and site hospital admission related to diabetes have shown significant associations with self-care practice. Those variables were included in a multivariable regression analysis and this showed age, income, marital status and poor knowledge about diabetes were found to be significantly associated with self-care practice.
Table 2 Clinical Characteristics and Lifestyle Related Information of Diabetic Patients in ACSH, Tigray, Ethiopia, $2015(\mathrm{~N}=410)$

\begin{tabular}{|c|c|c|c|}
\hline \multicolumn{2}{|l|}{ Variable } & \multirow{3}{*}{$\begin{array}{l}\text { Frequency } \\
193 \\
217\end{array}$} & \multirow{3}{*}{$\begin{array}{l}\text { Percentage } \\
47.1 \\
52.9\end{array}$} \\
\hline Type of Diabetes & Type I & & \\
\hline & Type 2 & & \\
\hline Duration of & $<5$ years & 290 & 70.7 \\
\hline Diabetes & $\begin{array}{l}5 \text { and above } \\
\text { years }\end{array}$ & 120 & 29.3 \\
\hline \multirow[t]{2}{*}{ Family history } & Yes & 391 & 95.4 \\
\hline & No & 19 & 4.6 \\
\hline \multirow[t]{4}{*}{ FBS } & $70-140$ & 181 & 44.1 \\
\hline & $140-180$ & 90 & 22 \\
\hline & $180-250$ & 98 & 23.9 \\
\hline & $>250$ and $<70$ & 41 & 10 \\
\hline \multirow[t]{3}{*}{ BMI } & $<18.5$ & 45 & 11 \\
\hline & $18.5-24.9$ & 299 & 72.9 \\
\hline & $>24.9$ & 66 & 16.1 \\
\hline \multirow[t]{2}{*}{ Physical Exercise } & Yes & 247 & 60.2 \\
\hline & No & 163 & 39.8 \\
\hline \multirow[t]{3}{*}{ Smoking } & Yes & 2 & 0.5 \\
\hline & No & 402 & 98 \\
\hline & Quit & 6 & 1.5 \\
\hline
\end{tabular}

Participants aged $>30$ years had 1.577 higher odds of poor self-care activities and participants earning an income of $>15,000$ birr per year were had $48 \%$ less chance of poor self-care activities. From this study, married participants were 2.2 times more likely to practice poor self-care activities compared with single status participants. Respondents with poor knowledge were 5 times more likely to have poor self-care activities compared with participants with good knowledge (Table S2).

\section{Discussion}

This study evaluated associated factors and self-care practice in adult diabetic patients on follow-up in ACSH. Just over half $(50.5 \%)$ of study subjects were found to be practicing poor self-care. This figure is lower than a study done in Jimma University Specialized Teaching Hospital and Harari, both in Ethiopia, of which 55\% and $60.7 \%$ of the participants had poor self-care practice, respectively. ${ }^{3,10}$ Another study done in Kenya revealed that $59 \%$ of the participants had poor self-care practice. ${ }^{11}$ This difference could be due to variation in sample size, socioeconomic and educational background. 
This figure is relatively higher than a study done in Nekemte Referral Hospital in which poor self-care practice was reported to be $45 \%{ }^{6}$ and a study done from Tikur Anbessa specialized hospital in which they found that $44 \%$ of participants did not adhere to overall self-care practice. ${ }^{12}$ This result was also higher than studies done in Iran and Finland, in which $26 \%$ and $19 \%$ of respondents, respectively did not adhere to overall self-care practice. $^{13,14}$ This big discrepancy might be because of lack of awareness on the importance of all the activities of diabetic self-care by the patient, lifestyle differences, and cultural and socioeconomic variations. This study is similar to a study done among diabetic patients on followup at public health centers and private clinics in Addis Ababa where the magnitude of poor diabetes self-care practice was $47.7 \%{ }^{15}$

In this study participants with a relatively high average annual income (>15,000 ETB) were found to have $48 \%$ less chance of poor self-care activities. This is similar to a study done in Harari and Nekmet Referral Hospital, ${ }^{6,10}$ which might be explained by the fact that patients with a relatively high income can easily access the healthy lifestyle recommended for diabetic patients (e.g., adherence to diabetic diet and exercise) and they can afford their medication and home glucose monitoring.

Poor knowledge overall on diabetes was found to have a statistically significant association with poor self-care activities. This study showed 144 (34.6\%) participants had poor knowledge. This was lower than a study done in Nekemte Referral Hospital which reported $45.7 \%$ and a study done in public and private primary level health care in Addis Ababa which reported $42.5 \%$ of respondents had poor knowledge on diabetes and self-care activities. $^{6,15}$ The possible explanation for the difference among these institutions may be parameters used to assess knowledge on diabetes. Another study conducted in Kenya reported that $72.8 \%$ of participants had poor knowledge ${ }^{11}$ and a study done in Egypt showed that the overall rate of inadequate knowledge regarding diabetes among participants was $47.7 \%{ }^{16}$ There were studies done in India which had a report of a high burden of poor diabetes knowledge. ${ }^{17}$ This difference could be due to the variation in assessment parameters, and differences in sociodemography.

\section{Strength and Limitation of the Study}

Systematic sample random sampling was used and this might reduce the bias. Amongst the notable limitations are self-reporting which might have introduced social desirability bias and under-reporting of some lifestyle behaviors such as alcohol consumption and cigarette smoking.

\section{Conclusion}

This study has revealed that $50.5 \%$ of study participants had poor self-care practice. Statistically significant associated factors of poor self-care practice in this study were low income and poor knowledge about DM.

\section{Recommendation}

The Federal Ministry of Health and Regional Health bureaus in collaboration with ACSH should invest in promoting patient awareness about diabetes and its related chronic complications, using a number of differentinformation dissemination tools aimed at the whole community. The diabetic unit has to identify patients with poor economic status and introduce a system to provide medication free of charge for low income patients. The reason for poor knowledge must be clear in future studies.

\section{Abbreviations}

AADE, American Association of Diabetes Educators; ADA, American Diabetes Association; ACSH, Ayder Comprehensive Specialized Hospital; DM, diabetic mellitus; FBS, fasting blood sugar; GTT, glucose tolerance test; IDF, International Diabetes Federation; RBS, random blood sugar; WHO, World Health Organization.

\section{Data Sharing Statement}

Please contact Migbnesh Weledegebriel for data requests.

\section{Ethical Consideration}

Ethical clearance was obtained from the Institutional Review Board (IRB) of the College of Health Sciences of Mekelle University with Ethical Review Committee (ERC) number of 0549/2015 and Informed consent for Participation was obtained from all respondents. We confirm that the study was conducted in accordance with the declaration of Helsinki.

\section{Acknowledgments}

We would like to thank our study participants for their willingness to participate, our data collectors and the DM clinic staff. Special thanks to Dr. Hansa Haftu for encouraging and helping find this journal to submit our work. We would like to acknowledge Mekelle University, College of 
Health Sciences, Department of Internal Medicine for giving us this opportunity.

\section{Author Contributions}

All authors made a significant contribution to the work reported in the conception, study design, execution, acquisition of data, analysis and interpretation, or in all these areas; took part in drafting, revising or critically reviewing the article; gave final approval of the version to be published; have agreed on the journal to which the article has been submitted; and agree to be accountable for all aspects of the work.

\section{Funding}

No specific funding was received for this study.

\section{Disclosure}

The authors report no conflicts of interest in this work.

\section{References}

1. Federation ID. International Diabetes Federation: Diabetes Atlas. 4th ed. 2009.

2. Federation ID. International Diabetes Federation: IDF Diabetes Atlas. 2013.

3. Yemane T, Belachew T, Asaminew BBO. Type 2 diabetes mellitus in Jimma Town SouthwestEthiopia. Ethiopia J Health Sci. 2007;17 (2): 107 .

4. Abdulkadir JRA, Reja A. Management of diabetes Mellitus; coping with limited facilities. Ethiop Med J. 2001;39(4):349-365.

5. Heinrich E. Self-management interventions for type2 diabetes: a systematic review. EDN. 2010;7(2).
6. Amente T, Belachew T, Hailu E, Berhanu N. Self care practices and it's predictors among diabetes mellitus patients on follow up. World J Med Med Res. 2014;2(3):1-16.

7. Fsc HT. level and associated factors with knowledge and practice of diabetes mellitus. Clin Med Res. 2013;2(6):110-120. doi:10.11648/j. cmr.20130206.11

8. Group UKPDS (UKPDS). Intensive blood- glucose control with sulphonylureas or insulin compared with conventional treatment and risk of complications in patients with type 2 diabetes (UKPDS 33). Lancet. 1998;352(9131):837-853. doi:10.1016/S0140-6736(98) 07019-6

9. Association AD. Standards of medical care in diabetes. Diabetes Care. 2007.

10. Ayele K, Tesfa B. Self care behavior among patients with diabetes in Harari, Eastern Ethiopia. Health Belief Model Perspect. 2012;7(4).

11. William K, Zachary M. Knowledge, attitude and practices related to diabetes among community members in four provinces in Kenya: a Cross-Sectional Study. Afr J Prm Heal Care Fam Med. 2010;4(1).

12. Berhe KK, Demissie A, Kahsay AB, Gebru HB. Diabetes self care practices and associated factors among type 2 diabetic patients. Int J Pharm Sci Res. 2012;3(11):4219-4229.

13. Al ZY. Assessment of self-care practice and its associated factors among diabetic patients. J Res Health Sci. 2011;11(1):33 38.

14. Hentinen MT, Hentinen M. Adherence to self-care and glycaemic control among people with insulin-dependent diabetes mellitus. $J A d v$ Nurs. 2001;34(6):780-786. doi:10.1046/j.1365-2648.2001.01808.x

15. Tassew B. Assessment of Diabetes Self-Care Practice and Its Associated Factors Among Patient on Follow Up at Public and Private Primary Level Health Care in Addis Ababa. AAU Institutional Repository. 2015.

16. Ghada El-Khawaga MD, Farida Abdel-Wahab M. Knowledge, attitudes, practice and compliance of diabetic patients in Dakahlia, Egypt. Eur J Res Med Sci. 2015;3(1).

17. Basu S, Garg S, Sharma N, Singh MM, Garg S. Adherence to self-care practices, glycemic status and influencing factors in diabetes patients in a tertiary care hospital in Delhi. World J Diabetes. 2018;9 (5):72-79.

\section{Publish your work in this journal}

Diabetes, Metabolic Syndrome and Obesity: Targets and Therapy is an international, peer-reviewed open-access journal committed to the rapid publication of the latest laboratory and clinical findings in the fields of diabetes, metabolic syndrome and obesity research. Original research, review, case reports, hypothesis formation, expert opinion and commentaries are all considered for publication. The manuscript management system is completely online and includes a very quick and fair peer-review system, which is all easy to use. Visit http://www.dovepress.com/testimonials.php to read real quotes from published authors. 